\title{
CONVERGENCE ANALYSIS FOR THE MULTIPLICATIVE SCHWARZ PRECONDITIONED INEXACT NEWTON ALGORITHM*
}

\author{
LULU LIU ${ }^{\dagger}$ AND DAVID E. KEYES ${ }^{\dagger}$
}

\begin{abstract}
The multiplicative Schwarz preconditioned inexact Newton (MSPIN) algorithm, based on decomposition by field type rather than by subdomain, was recently introduced to improve the convergence of systems with unbalanced nonlinearities. This paper provides a convergence analysis of the MSPIN algorithm. Under reasonable assumptions, it is shown that MSPIN is locally convergent, and desired superlinear or even quadratic convergence can be obtained when the forcing terms are picked suitably.
\end{abstract}

Key words. nonlinear equations, nonlinear preconditioning, multiplicative Schwarz, local convergence

AMS subject classifications. $65 \mathrm{H} 10,65 \mathrm{H} 20,65 \mathrm{~N} 12,65 \mathrm{~N} 22$

DOI. $10.1137 / 15 \mathrm{M} 1028182$

1. Introduction. Globalized Newton-like methods are widely used in solving nonlinear algebraic systems. However, their performance can be degraded by "nonlinear stiffness," which results in a considerable number of unproductive iterations, in terms of global residual norm reduction, as some controlling feature (e.g., boundary or interior layer, aerodynamic shock, reaction zone) of the solution evolves. During this stiff phase, the global Newton updates, which may require considerable linear algebraic effort to obtain, change very little except in the controlling components. The additive Schwarz preconditioned inexact Newton method (ASPIN) [2] was devised to attack such "unbalanced nonlinearities" and improve global convergence properties. Given the discrete nonlinear function $F: R^{n} \rightarrow R^{n}$, we want to find a vector $x^{*} \in R^{n}$ such that

$$
F\left(x^{*}\right)=0,
$$

where $F(x)=\left[F_{1}(x), F_{2}(x), \ldots, F_{n}(x)\right]^{T}$, and $x=\left[x_{1}, x_{2}, \ldots, x_{n}\right]^{T}$. ASPIN transforms $F(x)$ into a new nonlinear system $\mathcal{F}(x)$ with the same root, which is solved by a Jacobian-free [12] inexact Newton method. It has been shown in applications $[2,3,4,10,19,20]$ that ASPIN can be effective in reducing the number of global outer Newton iterations for the preconditioned system, relative to the original. The expense of the preconditioning is in the solution of relatively rapidly convergent and potentially asynchronously executed inner Newton iterations over smaller sets of unknowns. The savings can be substantial and the reduction of globally synchronizing Newton and inner Krylov iterations is favorable for emerging computing architectures.

The ASPIN algorithm was introduced in [2] as a nonlinear domain decomposition method, though its algebraic generalization to other types of decompositions was anticipated. Motivated by partitioning of degrees of freedom by field type, the

* Received by the editors July 6, 2015; accepted for publication (in revised form) August 16, 2016; published electronically October 26, 2016.

http://www.siam.org/journals/sinum/54-5/M102818.html

Funding: This work was supported by the Extreme Computing Research Center at KAUST and by the Aramco KAUST Master Research Agreement ORS 1438.

${ }^{\dagger}$ Program in Applied Mathematics and Computational Science and Center for Extreme Computing, King Abdullah University of Science and Technology, Thuwal 23955-6900, Saudi Arabia (lulu.liu@kaust.edu.sa, david.keyes@kaust.edu.sa). 


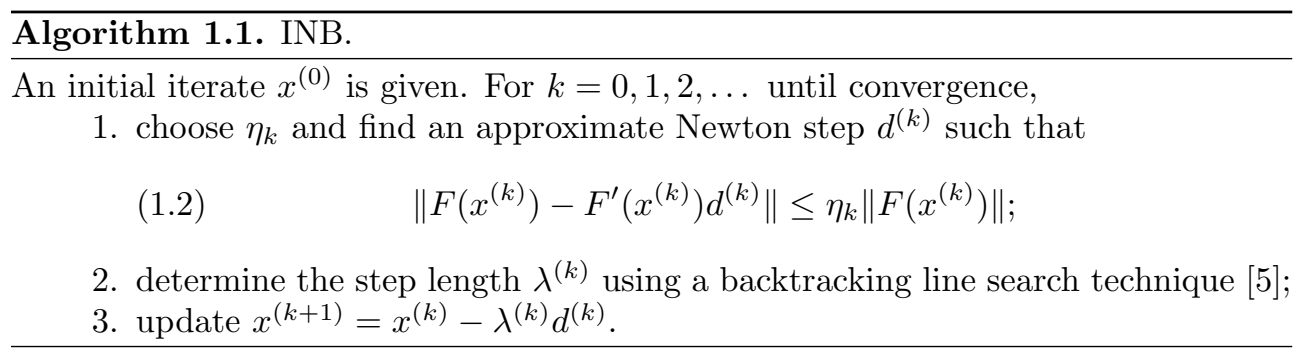

multiplicative Schwarz preconditioned inexact Newton (MSPIN) algorithm [13], was introduced as a Gauss-Seidel-like variant of ASPIN. Typically, the additive form of preconditioning is applied in a domain-split manner to generate massive concurrency that scales with mesh resolution, whereas the multiplicative, being sequential, is applied in a field-split manner over a small fixed number of partitions. Numerical examples in [13] illustrate that field-split MSPIN can restructure strong nonlinearities and improve the robustness and convergence rate of Newton prior to or even in the absence of other globalization strategies, such as continuation in parameters, in mesh resolution, or in pseudotime. Intuitively, nonlinear preconditioning should expand the region in which the contours of the merit function for Newton have hyperellipsoidal structure. A simple two-dimensional illustration, in which a polynomial system is reduced from ninth-order to third-order by nonlinear preconditioning, is given in section 3.2 of [13].

Some preliminary convergence analysis of ASPIN was carried out in the context of semilinear elliptic PDEs in [14]. Theoretical support for the local convergence of additive nonlinear preconditioning at up to a quadratic rate, for suitable assumptions, was provided in [1]. The convergence analysis of the multiplicative version of nonlinear preconditioning has not yet been given. In this paper, it is shown that MSPIN is locally convergent and that the convergence rate is superlinear or quadratic under suitable assumptions. The results are similar to those obtained in [1] for the additive variant, and tools similar to those in $[1,9]$ are employed.

The inexact Newton method with backtracking (INB) $[5,7,11,16]$ is the fundamental component of both ASPIN and MSPIN, so the INB algorithm is summarized in Algorithm 1.1.

The term $\eta_{k} \in[0,1)$, referred to as the "forcing term," reflects how accurately we solve $F^{\prime}\left(x^{(k)}\right) d^{(k)}=F\left(x^{(k)}\right)$ using a Krylov subspace method, such as GMRES $[17,18]$. Eisenstat and Walker suggested two strategies for choosing $\eta_{k}$ in [8]. The parameter $\lambda^{(k)}$ determines how far it goes along the inexact Newton direction $d^{(k)}$. The backtracking line search procedure for choosing $\lambda^{(k)}$ is described in section 2.2.

The rest of the paper is organized as follows. In section 2 , we briefly review the MSPIN algorithm and list some properties. Section 3 contains a proof of local convergence of MSPIN under some reasonable conditions. The convergence rate is discussed in section 4 , and we conclude in section 5 .

2. MSPIN. We consider a nonlinear root-finding problem (1.1) and assume that the function $F(x)$ is continuously differentiable. In the MSPIN algorithm, the nonlinear function $F(x)$ is split conformally into $2 \leqslant N \leqslant n$ nonoverlapping components representing distinct physical features as

$$
F(x)=F\left(u_{1}, \ldots, u_{N}\right)=\left[\begin{array}{c}
\hat{F}_{1}\left(u_{1}, \ldots, u_{N}\right) \\
\vdots \\
\hat{F}_{N}\left(u_{1}, \ldots, u_{N}\right)
\end{array}\right]=0,
$$


where $x=\left[x_{1}, \ldots, x_{n}\right]^{T}=\left[u_{1}, \ldots, u_{N}\right]^{T} \in R^{n} ; u_{i}$ and $\hat{F}_{i}$ denote subpartitions of $x$ and $F$, respectively, $i=1, \ldots, N$. In the MSPIN algorithm, the submodels are solved sequentially for the physical variable corrections, and the preconditioned system whose root is to be found consists of the sum of these corrections.

The multiplicative Schwarz preconditioned function

$$
\mathcal{F}(x)=\left[\begin{array}{c}
T_{1}\left(u_{1}, \ldots, u_{N}\right) \\
\vdots \\
T_{N}\left(u_{1}, \ldots, u_{N}\right)
\end{array}\right]
$$

is obtained by solving the following equations:

$$
\begin{aligned}
& \hat{F}_{1}\left(u_{1}-T_{1}(x), u_{2}, u_{3}, \ldots, u_{N}\right)=0, \\
& \hat{F}_{2}\left(u_{1}-T_{1}(x), u_{2}-T_{2}(x), u_{3}, \ldots, u_{N}\right)=0, \\
& \quad \vdots \\
& \hat{F}_{N}\left(u_{1}-T_{1}(x), u_{2}-T_{2}(x), u_{3}-T_{3}(x), \ldots, u_{N}-T_{N}(x)\right)=0 .
\end{aligned}
$$

As with the ASPIN algorithm in [2], the MSPIN method solves the global preconditioned problem in (2.2) using the INB algorithm.

2.1. Some properties. We discuss key properties of the MSPIN algorithm under reasonable assumptions. Specifically, we prove that the preconditioned function $\mathcal{F}(x)$ is continuously differentiable and we give a formula for the Jacobian $\mathcal{F}^{\prime}(x)$.

Let

$$
S=\{1, \ldots, n\}
$$

be an index set, and

$$
F(x)=\left[F_{1}(x), F_{2}(x), \ldots, F_{n}(x)\right]^{T}=\left[\hat{F}_{1}(x), \hat{F}_{2}(x), \ldots, \hat{F}_{N}(x)\right]^{T},
$$

where $\hat{F}_{i}(x)=\hat{F}_{i}\left(u_{1}, \ldots, u_{N}\right)$ is defined in (2.1). We define $S_{i}=\left\{i_{1}, i_{2}, \ldots, i_{n_{i}}\right\} \subset S$ as

$$
S_{i}=\left\{j \mid F_{j}(x) \text { belongs to the function } \hat{F}_{i}(x), j \in S\right\},
$$

where $i=1,2, \ldots, N$, and $n_{1}+n_{2}+\cdots+n_{N}=n$. We define a set of restriction matrices $R_{i} \in R^{n_{i} \times n}$ as

$$
\left(R_{i}\right)_{k, l}=\left\{\begin{array}{lc}
1, & l=i_{k} \\
0 & \text { otherwise }
\end{array}\right.
$$

and then we define

$$
E_{i}=R_{i}^{T} R_{i} \in R^{n \times n}, \quad i=1, \ldots, N .
$$

It is clear that $F(x)=\sum_{i=1}^{N} R_{i}^{T} \hat{F}_{i}(x)$.

Illustrating with $n=3, N=2$, let $S_{1}=\{1\}, S_{2}=\{2,3\}, n_{1}=1$, and $n_{2}=2$, to get

$$
\hat{F}_{1}(x)=F_{1}\left(u_{1}, u_{2}\right), \quad \hat{F}_{2}(x)=\left[\begin{array}{c}
F_{2}\left(u_{1}, u_{2}\right) \\
F_{3}\left(u_{1}, u_{2}\right)
\end{array}\right],
$$

Copyright $@$ by SIAM. Unauthorized reproduction of this article is prohibited. 
where $x=\left[x_{1}, x_{2}, x_{3}\right]^{T}, u_{1}=x_{1}, u_{2}=\left[x_{2}, x_{3}\right]^{T}$.

$$
\begin{gathered}
R_{1}=[1,0,0], \quad R_{2}=\left[\begin{array}{lll}
0 & 1 & 0 \\
0 & 0 & 1
\end{array}\right], \\
E_{2}=\left[\begin{array}{lll}
1 & 0 & 0 \\
0 & 0 & 0 \\
0 & 0 & 0
\end{array}\right], \quad E_{2}=\left[\begin{array}{lll}
0 & 0 & 0 \\
0 & 1 & 0 \\
0 & 0 & 1
\end{array}\right] .
\end{gathered}
$$

For the nonlinear problem (2.1), we make the following assumptions for $F(x)$.

Assumption 2.1. The function $F(x)$ is well defined in a neighborhood $U$ of the exact solution $x^{*}$ :

(i) The Jacobian

$$
F^{\prime}(x)=J(x)=\left[\begin{array}{ccccc}
\frac{\partial \hat{F}_{1}}{\partial u_{1}} & \frac{\partial \hat{F}_{1}}{\partial u_{2}} & \frac{\partial \hat{F}_{1}}{\partial u_{3}} & \cdots & \frac{\partial \hat{F}_{1}}{\partial u_{N}} \\
\frac{\partial \hat{F}_{2}}{\partial u_{1}} & \frac{\partial \hat{F}_{2}}{\partial u_{2}} & \frac{\partial \hat{F}_{2}}{\partial u_{3}} & \cdots & \frac{\partial \hat{F}_{2}}{\partial u_{N}} \\
\vdots & \vdots & \vdots & & \vdots \\
\frac{\partial \hat{F}_{N}}{\partial u_{1}} & \frac{\partial \hat{F}_{N}}{\partial u_{2}} & \frac{\partial \hat{F}_{N}}{\partial u_{3}} & \cdots & \frac{\partial \hat{F}_{N}}{\partial u_{N}}
\end{array}\right]
$$

is continuous in $U$ and $J\left(x^{*}\right)$ is nonsingular.

(ii) The submatrices $R_{i} J\left(x^{*}\right) R_{i}^{T}$ are invertible, $i=1,2, \ldots, N$.

We now recall results from Lemma 4.3 and Theorem 4.5 in [13] in the following lemma. It guarantees that $\mathcal{F}(x)$ in $(2.2)$ is well defined and continuous and shows the equivalence of two nonlinear systems (2.1) and (2.2).

Lemma 2.2. Under Assumption 2.1, there exists a neighborhood $D_{1} \subset U$ of the exact solution $x^{*}$ such that the following hold:

(i) The subproblems in (2.3) are all uniquely solvable. There is a unique continuous function $T_{i}(x)$ such that (2.3) hold for any $x \in D_{1}$ with $T_{i}\left(x^{*}\right)=0$, $i=1,2, \ldots, N$.

(ii) The nonlinear systems (2.1) and (2.2) have the same solution in $D_{1}$.

We define

$$
D F(x, y)=\int_{0}^{1} J(x+t(y-x)) d t
$$

and $\tilde{T}_{i} \in R^{n}$ is defined as

$$
\tilde{T}_{0}(x)=\mathbf{0} \in R^{n} \quad \forall x \in D_{1},
$$

and

$$
\tilde{T}_{1}(x)=\left[\begin{array}{c}
T_{1}(x) \\
0 \\
\vdots \\
0
\end{array}\right], \quad \tilde{T}_{i}(x)=\left[\begin{array}{c}
T_{1}(x) \\
\vdots \\
T_{i}(x) \\
\mathbf{0}_{N-i}
\end{array}\right], \quad \tilde{T}_{N}(x)=\left[\begin{array}{c}
T_{1}(x) \\
T_{2}(x) \\
\vdots \\
T_{N}(x)
\end{array}\right]
$$

Copyright $@$ by SIAM. Unauthorized reproduction of this article is prohibited. 
for any $x \in D_{1}$, where $\mathbf{0}_{N-i} \in R^{N-i}$ is a zero vector, $i=2, \ldots, N-1$. From (i) in Lemma 2.2 , it is seen that

$$
\tilde{T}_{i}(x)=\sum_{k=1}^{i} R_{k}^{T} T_{k}(x)
$$

is continuous and $\tilde{T}_{i}(x) \rightarrow 0$ as $x \rightarrow x^{*}, i=1,2, \ldots, N$.

Following [9], the multiplicative Schwarz preconditioned function $\mathcal{F}(x)$ in $(2.2)$ is also written as

$$
\mathcal{F}(x)=\mathcal{T}_{1}(x)+\sum_{i=2}^{N} \mathcal{T}_{i}\left(x-\tilde{T}_{i-1}(x)\right),
$$

where

$$
\mathcal{T}_{i}\left(x-\tilde{T}_{i-1}(x)\right)=R_{i}^{T} T_{i}(x) .
$$

Due to continuity on $T_{i}$ in a neighborhood $D_{1}$ from Lemma 2.2, it follows from [6, 9] that we have

$$
\mathcal{T}_{i}\left(x^{\prime}\right)-\mathcal{T}_{i}\left(x^{\prime \prime}\right)=D \mathcal{T}_{i}\left(x^{\prime}, x^{\prime \prime}\right)\left(x^{\prime}-x^{\prime \prime}\right) \quad \forall x^{\prime}, x^{\prime \prime} \in D_{1},
$$

where $D \mathcal{T}_{i}\left(x^{\prime}, x^{\prime \prime}\right)$ is defined as

$$
D \mathcal{T}_{i}\left(x^{\prime}, x^{\prime \prime}\right)=R_{i}^{T}\left[R_{i} D F R_{i}^{T}\right]^{-1} R_{i} D F, \quad D F=D F\left(x^{\prime}-\mathcal{T}_{i}\left(x^{\prime}\right), x^{\prime \prime}-\mathcal{T}_{i}\left(x^{\prime \prime}\right)\right) .
$$

Remark 2.3. We make the right-to-left operator convention:

$$
\prod_{i=1}^{N} A_{i}=A_{N} \cdots A_{2} A_{1} .
$$

Theorem 1.7 and Remark 1.8 in [9] show that

$$
\begin{aligned}
& \mathcal{F}\left(x^{\prime}\right)-\mathcal{F}\left(x^{\prime \prime}\right) \\
& \quad=\left(I-\prod_{i=1}^{N}\left[I-D \mathcal{T}_{i}\left(x^{\prime}-\tilde{T}_{i-1}\left(x^{\prime}\right), x^{\prime \prime}-\tilde{T}_{i-1}\left(x^{\prime \prime}\right)\right)\right]\right)\left(x^{\prime}-x^{\prime \prime}\right)
\end{aligned}
$$

for any $x^{\prime}, x^{\prime \prime} \in D_{1}$.

Note that $z=x-\tilde{T}_{i-1}(x)$, and then using (2.15) and (2.17) we have

$$
\begin{aligned}
z-\mathcal{T}_{i}(z) & =x-\tilde{T}_{i-1}(x)-\mathcal{T}_{i}\left(x-\tilde{T}_{i-1}(x)\right) \\
& =x-\tilde{T}_{i-1}(x)-R_{i}^{T} T_{i}(x) \\
& =x-\tilde{T}_{i}(x)
\end{aligned}
$$

and it follows from (2.19) and (2.21) that

$$
\begin{aligned}
& \mathcal{F}\left(x^{\prime}\right)-\mathcal{F}\left(x^{\prime \prime}\right) \\
= & \left(I-\prod_{i=1}^{N}\left(I-Q_{i}\left(x^{\prime}, x^{\prime \prime}\right)\right)\right)\left(x^{\prime}-x^{\prime \prime}\right) \quad \text { for } x^{\prime}, x^{\prime \prime} \in D_{1},
\end{aligned}
$$

where $Q_{i}\left(x^{\prime}, x^{\prime \prime}\right)$ is defined as

$$
R_{i}^{T}\left[R_{i} D F\left(x^{\prime}-\tilde{T}_{i}\left(x^{\prime}\right), x^{\prime \prime}-\tilde{T}_{i}\left(x^{\prime \prime}\right)\right) R_{i}^{T}\right]^{-1} R_{i} D F\left(x^{\prime}-\tilde{T}_{i}\left(x^{\prime}\right), x^{\prime \prime}-\tilde{T}_{i}\left(x^{\prime \prime}\right)\right) .
$$

Copyright $@$ by SIAM. Unauthorized reproduction of this article is prohibited. 
TheOrem 2.4. Let Assumption 2.1 hold. Then there is a neighborhood $D \subset U$ of the exact solution $x^{*}$ such that $\mathcal{F}(x)$ defined in $(2.2)$ is continuously differentiable. Moreover,

$$
\mathcal{F}^{\prime}(x)=I-\prod_{i=1}^{N}\left(I-W_{i}(x)\right), \quad x \in D,
$$

where $W_{i}(x)=R_{i}^{T}\left[R_{i} J\left(x-\tilde{T}_{i}(x)\right) R_{i}^{T}\right]^{-1} R_{i} J\left(x-\tilde{T}_{i}(x)\right)$.

Proof. Under Assumption 2.1, Lemma 2.2 shows that there exists a neighborhood $D_{1} \subset U$ of $x^{*}$ such that $\mathcal{F}(x)$ is continuous, and then (2.23) holds for any $x^{\prime}, x^{\prime \prime} \in$ $D_{1}$. Since $R_{i} J\left(x^{*}\right) R_{i}^{T}$ is invertible and $J(x)$ is continuous, by Lemma 2.3.3 in [15], there exists a neighborhood $D_{2} \subset U$ of $x^{*}$ such that $R_{i} J(x) R_{i}^{T}$ is invertible and $\left[R_{i} J(x) R_{i}^{T}\right]^{-1}$ is continuous in $D_{2}, i=1,2, \ldots, N$.

Let $D \subset D_{1} \cap D_{2} \subset U$ be a neighborhood of the exact solution $x^{*}$ such that $x-\tilde{T}_{i}(x) \in D_{2}$ for any $x \in D$ and $i=1,2, \ldots, N$, and then we define

$$
A(x)=I-\prod_{i=1}^{N}\left(I-W_{i}(x)\right), \quad x \in D
$$

where $W_{i}(x)=R_{i}^{T}\left[R_{i} J\left(x-\tilde{T}_{i}(x)\right) R_{i}^{T}\right]^{-1} R_{i} J\left(x-\tilde{T}_{i}(x)\right), i=1,2, \ldots, N$. Note that $A(x)$ is continuous in $D$. Due to the continuity of $J(x)$ and $\tilde{T}_{i}(x)$, we have

$$
D F\left(x+h-\tilde{T}_{i}(x+h), x-\tilde{T}_{i}(x)\right) \rightarrow J\left(x-\tilde{T}_{i}(x)\right) \quad \text { as } h \rightarrow 0 .
$$

Using (2.23) and (2.26), we have

$$
\lim _{\|h\| \rightarrow 0} \frac{\|\mathcal{F}(x+h)-\mathcal{F}(x)-A(x) h\|}{\|h\|}=0,
$$

which implies that $\mathcal{F}$ is Fréchet-differentiable and $\mathcal{F}^{\prime}(x)=A(x)$.

In [9], the expression in (2.25) of $\mathcal{F}^{\prime}(x)$ was employed to develop the damped Richardson scheme instead of the INB method as the solver of the preconditioned system (2.2). The following theorem implies that the formula (2.25) can be simplified to the form of multiplication of two matrices, and then the matrix-vector multiplication can be carried out instead of explicitly forming the full Jacobian matrix $\mathcal{F}^{\prime}(x)$ when we solve the Newton equation.

TheOrem 2.5. Let Assumption 2.1 hold, and let $D$ be the neighborhood determined in Theorem 2.4. Let $x=\left[u_{1}, u_{2}, \ldots, u_{N}\right]^{T}$, where $u_{i} \in R^{n_{i}}$ and $\delta_{i}=u_{i}-T_{i}(x)$, $i=1,2, \ldots, N$. Then $\mathcal{F}^{\prime}(x)$ from Theorem 2.4 has the form

$$
\mathcal{J}(x)=\left[\begin{array}{cccc}
\frac{\partial \hat{F}_{1}}{\partial \delta_{1}} & & & \\
\frac{\partial \hat{F}_{2}}{\partial \delta_{1}} & \frac{\partial \hat{F}_{2}}{\partial \delta_{2}} & & \\
\vdots & \vdots & \ddots & \\
\frac{\partial \hat{F}_{N}}{\partial \delta_{1}} & \frac{\partial \hat{F}_{N}}{\partial \delta_{2}} & \cdots & \frac{\partial \hat{F}_{N}}{\partial \delta_{N}}
\end{array}\right]^{-1}\left[\begin{array}{ccccc}
\frac{\partial \hat{F}_{1}}{\partial \delta_{1}} & \frac{\partial \hat{F}_{1}}{\partial u_{2}} & \frac{\partial \hat{F}_{1}}{\partial u_{3}} & \cdots & \frac{\partial \hat{F}_{1}}{\partial u_{N}} \\
\frac{\partial \hat{F}_{2}}{\partial \delta_{1}} & \frac{\partial \hat{F}_{2}}{\partial \delta_{2}} & \frac{\partial \hat{F}_{2}}{\partial u_{3}} & \cdots & \frac{\partial \hat{F}_{2}}{\partial u_{N}} \\
\vdots & \vdots & \vdots & & \vdots \\
\frac{\partial \hat{F}_{N}}{\partial \delta_{1}} & \frac{\partial \hat{F}_{N}}{\partial \delta_{2}} & \frac{\partial \hat{F}_{N}}{\partial \delta_{3}} & \cdots & \frac{\partial \hat{F}_{N}}{\partial \delta_{N}}
\end{array}\right]
$$

Copyright $@$ ㅇ by SIAM. Unauthorized reproduction of this article is prohibited. 
Proof. Let $\mathbf{0}_{u_{i}} \in R^{n_{i} \times n_{i}}$ be the zero matrix that has the same dimension as the $u_{i}$ block, $i=1,2, \ldots, N$. We define

$$
\mathcal{L}(x) \equiv\left[\begin{array}{c}
l_{1} \\
l_{2} \\
\vdots \\
l_{N}
\end{array}\right]=\left[\begin{array}{cccc}
\frac{\partial \hat{F}_{1}}{\partial \delta_{1}} & & & \\
\frac{\partial \hat{F}_{2}}{\partial \delta_{1}} & \frac{\partial \hat{F}_{2}}{\partial \delta_{2}} & & \\
\vdots & \vdots & \ddots & \\
\frac{\partial \hat{F}_{N}}{\partial \delta_{1}} & \frac{\partial \hat{F}_{N}}{\partial \delta_{2}} & \cdots & \frac{\partial \hat{F}_{N}}{\partial \delta_{N}}
\end{array}\right]
$$

and

$$
\mathcal{H}(x) \equiv\left[\begin{array}{c}
h_{1} \\
h_{2} \\
\vdots \\
h_{N}
\end{array}\right]=\left[\begin{array}{ccccc}
\mathbf{0}_{u_{1}} & \frac{\partial \hat{F}_{1}}{\partial u_{2}} & \frac{\partial \hat{F}_{1}}{\partial u_{3}} & \cdots & \frac{\partial \hat{F}_{1}}{\partial u_{N}} \\
& \mathbf{0}_{u_{2}} & \frac{\partial \hat{F}_{2}}{\partial u_{2}} & \ldots & \frac{\partial \hat{F}_{2}}{\partial u_{N}} \\
& & \ddots & \ddots & \vdots \\
& & & \mathbf{0}_{u_{N-1}} & \frac{\partial \hat{F}_{N-1}}{\partial u_{N}} \\
& & & & \mathbf{0}_{u_{N}}
\end{array}\right]
$$

where $l_{i} \in R^{n_{i} \times n}, h_{i} \in R^{n_{i} \times n}, i=1,2, \ldots, N$, and then we have

$$
\mathcal{L}(x)=\sum_{i=1}^{N} R_{i}^{T} l_{i}, \quad \mathcal{H}(x)=\sum_{i=1}^{N} R_{i}^{T} h_{i} .
$$

We define $I_{u_{k}} \in R^{n_{k} \times n_{k}}$ as the identity matrix that has the same dimension as the $u_{k}$ block, $1 \leqslant k \leqslant N, k \neq i$. Note that

$$
I-W_{i}(x)=\left[\begin{array}{ccccccc}
I_{u_{1}} & & & & & & \\
& \ddots & & & & & \\
& & I_{u_{i-1}} & & & & \\
w_{i 1} & \cdots & w_{i, i-1} & \mathbf{0}_{u_{i}} & w_{i, i+1} & \cdots & w_{i_{N}} \\
& & & & I_{u_{i+1}} & & \\
& & & & & \ddots & \\
& & & & & & I_{u_{N}}
\end{array}\right]
$$

where

$$
w_{i, k}= \begin{cases}-\left(\frac{\partial \hat{F}_{i}}{\partial \delta_{i}}\right)^{-1} \frac{\partial \hat{F}_{i}}{\partial \delta_{k}}, & k<i \\ -\left(\frac{\partial \hat{F}_{i}}{\partial \delta_{i}}\right)^{-1} \frac{\partial \hat{F}_{i}}{\partial u_{k}}, & k>i\end{cases}
$$

It is then verified that

$$
\begin{aligned}
l_{k}\left(I-W_{i}(x)\right) & =l_{k}, \quad k<i, \\
h_{k}\left(I-W_{i}(x)\right) & =h_{k}, \quad k>i, \\
l_{k}\left(I-W_{i}(x)\right) & =-h_{k}, \quad k=i,
\end{aligned}
$$

Copyright $@$ by SIAM. Unauthorized reproduction of this article is prohibited. 
from which we may deduce that

$$
\begin{aligned}
\mathcal{L}(x) & \prod_{i=1}^{N}\left(I-W_{i}(x)\right) \\
& =\left(\sum_{i=1}^{N} R_{i}^{T} l_{i}\right) \prod_{i=1}^{N}\left(I-W_{i}(x)\right) \\
& =\left(\sum_{i=1}^{N-1} R_{i}^{T} l_{i}-R_{N}^{T} h_{N}\right) \prod_{i=1}^{N-1}\left(I-W_{i}(x)\right) \\
& =\left(\sum_{i=1}^{N-2} R_{i}^{T} l_{i}-\sum_{i=N-1}^{N} R_{i}^{T} h_{i}\right) \prod_{i=1}^{N-2}\left(I-W_{i}(x)\right) \\
& =\left(R_{1}^{T} l_{1}-\sum_{i=2}^{N} R_{i}^{T} h_{i}\right)\left(I-W_{1}(x)\right) \\
& =-\sum_{i=1}^{N} R_{i}^{T} h_{i}=-\mathcal{H}(x),
\end{aligned}
$$

and it follows that

$$
\mathcal{L}(x)\left(I-\prod_{i=1}^{N}\left(I-W_{i}(x)\right)\right)=\mathcal{L}(x)+\mathcal{H}(x),
$$

which completes the proof.

Theorem 2.5 obtains in a different way the same formula of the Jacobian $\mathcal{F}^{\prime}(x)$ shown in [13]. In the practical implementation of MSPIN, it is more convenient to use the following approximate Jacobian:

$$
\mathcal{J}(x) \approx \hat{\mathcal{J}}(x)=L(\bar{x})^{-1} J(\bar{x}), \quad \bar{x}=\left[\delta_{1}, \delta_{2}, \ldots, \delta_{N-1}, u_{N}\right]^{T},
$$

where $\delta_{i}=u_{i}-T_{i}, i=1,2, \ldots, N-1$, and $L(x)$ is defined as

$$
L(x)=\left[\begin{array}{ccccc}
\frac{\partial \hat{F}_{1}}{\partial u_{1}} & & & & \\
\frac{\partial \hat{F}_{2}}{\partial u_{1}} & \frac{\partial \hat{F}_{2}}{\partial u_{2}} & & & \\
\vdots & \vdots & \ddots & & \\
\frac{\partial \hat{F}_{N}}{\partial u_{1}} & \frac{\partial \hat{F}_{N}}{\partial u_{2}} & \frac{\partial \hat{F}_{N}}{\partial u_{3}} & \ldots & \frac{\partial \hat{F}_{N}}{\partial u_{N}}
\end{array}\right],
$$

which is the lower triangular part of $J(x)$. Since $F^{\prime}(x)=J(x)$ is continuous and the $R_{i} J\left(x^{*}\right) R_{i}^{T}$ are invertible, we know that $L(x)$ is continuous and $L\left(x^{*}\right)$ is nonsingular. Lemma 2.3.3 in [15] shows that there exists a neighborhood $U^{\prime} \subset U$ of the exact solution $x^{*}$ such that $L(x)$ are invertible, and $L(x)^{-1}$ are continuous in the neighborhood $U^{\prime}$. Due to the continuity of $T_{i}(x)$ (see (i) in Lemma 2.2), we know that $\delta_{i}=u_{i}-T_{i}(x) \rightarrow u_{i}^{*}$ as $x \rightarrow x^{*}$, where $x^{*}=\left[u_{1}^{*}, u_{2}^{*}, \ldots, u_{N}^{*}\right]^{T}$ and $i=1,2, \ldots, N$. Therefore, the following is seen. 
Remark 2.6. There is a neighborhood $D^{\prime} \subset D$ of the exact solution $x^{*}$ such that $L(x)^{-1}, \mathcal{J}(x), \hat{\mathcal{J}}(x), \mathcal{J}(x)^{-1}$, and $\hat{\mathcal{J}}(x)^{-1}$ are continuous in $D^{\prime}$, and

$$
\lim _{x \rightarrow x^{*}} \mathcal{J}(x)=\lim _{x \rightarrow x^{*}} \hat{\mathcal{J}}(x)=\mathcal{J}\left(x^{*}\right) .
$$

2.2. The MSPIN algorithm. To discuss convergence properties, we describe in Algorithm 2.1 the complete MSPIN algorithm. Considering $N$ different physically motivated subsets, we split $F(x)$ into $\hat{F}_{1}(x), \hat{F}_{2}(x), \ldots, \hat{F}_{N}(x)$, and the unknowns inherit the same partition structure, $x=\left[u_{1}, u_{2}, \ldots, u_{N}\right]^{T}$. In the implementation of MSPIN in Algorithm 2.1, the most interesting part is how to determine the partition of the physical variables, i.e., the set of equations that belongs to $\hat{F}_{i}$ for each $i$, because the best choice is generally problem-specific. The basic objective is to segregate subsystems that retard global Newton convergence from the rest and to make these systems as small as possible. Often, the controlling components are those associated with the scalar residual components that are largest in magnitude. However, the best partitions could evolve during the course of the problem and no theory is currently in hand for this. Once the partitioning and the initial guess are given, together with the customization of the parameters, we move on to step 1.

In step 1, based on the current approximate solution $x^{(k)}=\left[u_{1}^{(k)}, \ldots, u_{N}^{(k)}\right]^{T}$, the solutions $T_{i}^{(k)}$ of the submodels $\hat{F}_{i}, i=1,2, \ldots, N$, forming the global residual of the transformed system, are determined and checked for convergence.

In step 2, an approximate Newton step is accepted provided that (2.43) is satisfied. Note that the global Jacobian system (2.42) is solved using GMRES with no additional linear preconditioning. The Jacobian formula (2.35) implies that nonlinear preconditioning automatically provides a block Gauss-Seidel linear preconditioning for the original unpreconditioned equation.

In step 3, we carry out a backtracking line search algorithm to determine the step length $\lambda^{(k)}$ along the inexact Newton direction $d^{(k)}$. The backtracking line search technique is based on the following merit function

$$
f(x) \equiv \frac{1}{2}\|\mathcal{F}(x)\|_{2}^{2},
$$

and $\lambda^{(k)}$ is picked such that

$$
f\left(x^{(k)}-\lambda^{(k)} d^{(k)}\right) \leqslant f\left(x^{(k)}\right)-\alpha \lambda^{(k)} \nabla f\left(x^{(k)}\right)^{T} d^{(k)} .
$$

Following [5], $\alpha$ is set quite small in practice, so that only a small decrease in function value is required.

An easy calculation gives

$$
\nabla f\left(x^{(k)}\right)=\mathcal{J}\left(x^{(k)}\right)^{T} \mathcal{F}\left(x^{(k)}\right),
$$

which is replaced by $\hat{\mathcal{J}}\left(x^{(k)}\right)^{T} \mathcal{F}\left(x^{(k)}\right)$ in a practical algorithm.

For details about the implementation of MSPIN, see [13].

3. Local convergence. In this section, we give a theoretical foundation for the MSPIN algorithm and show that the MSPIN algorithm is locally convergent. Unfortunately and unsurprisingly, it does not seem possible to carry out a global convergence analysis of MSPIN, because this algorithm and its properties shown in section 2 are based on the assumptions on $F(x)$ in the neighborhood of the exact solution $x^{*}$. 


\section{Algorithm 2.1. MSPIN.}

Specify the initial guess $x^{(0)}=\left[u_{1}^{(0)}, u_{2}^{(0)}, \ldots, u_{N}^{(0)}\right]^{T}$.

Set the parameters $\alpha \in(0,1), \eta_{\max } \in(0,1)$, and $0<\theta_{\min }<\theta_{\max }<1$.

for $k=0,1,2, \ldots$, until convergence, do

1. Compute the nonlinear residual $\mathcal{F}\left(x^{(k)}\right)$ by solving the submodels.

(a) Starting from the initial guess $T_{i, 0}^{(k)}=0, i=1,2, \ldots, N$, find $T_{1}^{(k)}, T_{2}^{(k)}, \ldots, T_{N}^{(k)}$ by solving the following subproblems sequentially:

$$
\begin{aligned}
& \hat{F}_{1}\left(u_{1}^{(k)}-T_{1}^{(k)}, u_{2}^{(k)}, u_{3}^{(k)}, \ldots, u_{N}^{(k)}\right)=0, \\
& \hat{F}_{2}\left(u_{1}^{(k)}-T_{1}^{(k)}, u_{2}^{(k)}-T_{2}^{(k)}, u_{3}^{(k)}, \ldots, u_{N}^{(k)}\right)=0, \\
& \vdots \\
& \hat{F}_{N}\left(u_{1}^{k}-T_{1}^{(k)}, u_{2}^{(k)}-T_{2}^{(k)}, u_{3}^{(k)}-T_{3}^{(k)}, \ldots, u_{N}^{(k)}-T_{N}^{(k)}\right)=0 .
\end{aligned}
$$

(b) Form the global residual

$$
\mathcal{F}\left(x^{(k)}\right)=\left[\begin{array}{c}
T_{1}^{(k)} \\
T_{2}^{(k)} \\
\vdots \\
T_{N}^{(k)}
\end{array}\right] .
$$

(c) Check the stopping conditions on $\mathcal{F}\left(x^{(k)}\right)$.

2. Choose $\eta_{k}$ and find the inexact Newton direction $d^{(k)}$ by approximately solving

$$
\hat{\mathcal{J}}\left(x^{(k)}\right) d^{(k)}=\mathcal{F}\left(x^{(k)}\right),
$$

such that

$$
\left\|\mathcal{F}\left(x^{(k)}\right)-\hat{\mathcal{J}}\left(x^{(k)}\right) d^{(k)}\right\| \leq \eta_{k}\left\|\mathcal{F}\left(x^{(k)}\right)\right\|,
$$

where $\hat{\mathcal{J}}$ has the form $(2.35)$, and the forcing term $\eta_{k} \in\left[0, \eta_{\text {max }}\right]$.

3. Determine the step length $\lambda^{(k)}$ along the direction $d^{(k)}$.

Set $\lambda^{(k)}=1$

while $f\left(x^{(k)}-\lambda^{(k)} d^{(k)}\right)>f\left(x^{(k)}\right)-\alpha \lambda^{(k)} \mathcal{F}\left(x^{(k)}\right)^{T} \hat{\mathcal{J}}\left(x^{(k)}\right) d^{(k)}$ do choose some $\theta \in\left[\theta_{\min }, \theta_{\max }\right]$

$\lambda^{(k)}=\theta \lambda^{(k)}$

end while

4. Compute the new approximate solution.

$$
x^{(k+1)}=x^{(k)}-\lambda^{(k)} d^{(k)}
$$

end for

Let $N(x, r)=\{y \mid\|y-x\|<r\}$ represent an open ball with the center $x$ and a radius $r$, and $\|\cdot\|=\|\cdot\|_{2}$ for all the matrices and vectors in the remainder of the paper. The main assumptions are as follows.

Copyright (c) by SIAM. Unauthorized reproduction of this article is prohibited. 
Assumption 3.1. Assumption 2.1 holds, and we define

$$
K \equiv \max \left\{\left\|\mathcal{J}\left(x^{*}\right)\right\|,\left\|\mathcal{J}\left(x^{*}\right)^{-1}\right\|\right\}
$$

(a) In step 1(a) of Algorithm 2.1, we solve the subproblems exactly for $T_{i}^{(k)}$, for each $k$.

(b) In step 3 of Algorithm 2.1, we assume $\alpha$ is small enough so that

$$
\alpha \leqslant \frac{1-\eta_{\max }}{64 K^{4}\left(3+\eta_{\max }\right)} .
$$

(c) There exists a fixed small number $r>0$ such that $N\left(x^{*}, r\right) \subset D$, where $D$ is the neighborhood determined in Lemma 2.2. For any $x \in N\left(x^{*}, r\right)$, we further assume that

$\left(\mathrm{c}_{1}\right) L(x)^{-1}, \mathcal{J}(x), \hat{\mathcal{J}}(x), \mathcal{J}(x)^{-1}$ and $\hat{\mathcal{J}}(x)^{-1}$ are continuous;

$\left(\mathrm{c}_{2}\right)\|\mathcal{J}(x)\| \leqslant 2 K,\left\|\mathcal{J}(x)^{-1}\right\| \leqslant 2 K$, where $\mathcal{J}$ has the form (2.29);

$\left(c_{3}\right)\|\hat{\mathcal{J}}(x)\| \leqslant 2 K,\left\|\hat{\mathcal{J}}(x)^{-1}\right\| \leqslant 2 K$, where $\hat{\mathcal{J}}$ has the form (2.35);

$\left(c_{4}\right)\|\mathcal{J}(x)-\hat{\mathcal{J}}(x)\| \leqslant \frac{1-\eta_{\max }}{4 K\left(1+\eta_{\max }\right)} ;$

$\left(c_{5}\right)\left\|\mathcal{F}(x)-\mathcal{F}\left(x^{*}\right)-\mathcal{J}\left(x^{*}\right)\left(x-x^{*}\right)\right\| \leqslant \frac{1}{2 K}\left\|x-x^{*}\right\|$.

In (a), we assume that all of the subproblems are solved exactly, since introduction of the secondary iteration makes the discussion more complicated.

In (b), the proofs of Lemmas 3.2 and 4.1 require the assumption (3.2) for $\alpha$, and more details can be found in [1].

In (c), we can find a number $r_{1}>0$ from Remark 2.6 such that $\left(\mathrm{c}_{1}\right)-\left(\mathrm{c}_{4}\right)$ hold for any $x \in N\left(x^{*}, r_{1}\right) \subset D$. Theorem 2.4 shows that $\mathcal{F}(x)$ is continuously differentiable in a neighborhood of $x^{*}$, and hence we can find a number $r_{2}>0$ such that the last inequality $\left(\mathrm{c}_{5}\right)$ holds in $N\left(x^{*}, r_{2}\right) \subset D$ by Lemma 3.2 .10 in [15]. Let $r=\min \left\{r_{1}, r_{2}\right\}$, and $N\left(x^{*}, r\right)$ is the open ball required in (c). Appendix A gives an example that satisfies assumptions $\left(c_{1}\right)-\left(c_{5}\right)$.

Note that a line search procedure is carried out with initialization $\lambda^{(k)}=1$ in step 3 of Algorithm 2.1. Recalling results from Remark 3.1 and Theorem 3.5 in [1], we can obtain the following lemma. It shows that step 3 of Algorithm 2.1 will terminate the execution of the while-loop in a finite number of steps when the gradient of $f$ satisfies a mild condition. Moreover, there exists a positive lower bound for the resulting $\lambda^{(k)}$.

Lemma 3.2. Assume there exists $\gamma>0$ such that

$$
\|\nabla f(y)-\nabla f(z)\| \leqslant \gamma\|y-z\| \quad \forall y, z \in N\left(x^{*}, r\right) .
$$

Let $x \in N\left(x^{*}, \frac{r}{2}\right)$ satisfy $\mathcal{F}(x) \neq 0$ and $\|\mathcal{F}(x)\| \leqslant \frac{r}{8 K}$, and we find an inexact Newton direction $d \in R^{n}$ such that

$$
\|\mathcal{F}(x)-\hat{\mathcal{J}}(x) d\| \leq \eta\|\mathcal{F}(x)\|, \quad \eta \in\left[0, \eta_{\text {max }}\right] ;
$$

then it holds that $\mathcal{F}(x)^{T} \mathcal{J}(x) d>0$ and the while-loop in step 3 of Algorithm 2.1 will terminate in finite iterations and the determined step length $\lambda$ satisfies

$$
\lambda \geqslant \min \left\{1, \frac{\alpha \theta_{\min } \mathcal{F}(x)^{T} \mathcal{J}(x) d}{\gamma\|d\|^{2}}\right\} .
$$

Furthermore, we have $\|\mathcal{F}(x-\lambda d)\|<\|\mathcal{F}(x)\|$. 
LEMma 3.3. If $x \in N\left(x^{*}, r\right)$, then we have

$$
\left\|x-x^{*}\right\| \leqslant 2 K\|\mathcal{F}(x)\| .
$$

Proof. Since

$$
\left\|x-x^{*}\right\| \leqslant\left\|\mathcal{J}\left(x^{*}\right)^{-1}\right\|\left\|\mathcal{J}\left(x^{*}\right)\left(x-x^{*}\right)\right\|,
$$

by (3.1) and $\left(\mathrm{c}_{5}\right)$ in Assumption 3.1 and $\mathcal{F}\left(x^{*}\right)=0$, we have

$$
\begin{aligned}
\|\mathcal{F}(x)\| & \geqslant\left\|\mathcal{J}\left(x^{*}\right)\left(x-x^{*}\right)\right\|-\left\|\mathcal{F}(x)-\mathcal{F}\left(x^{*}\right)-\mathcal{J}\left(x^{*}\right)\left(x-x^{*}\right)\right\| \\
& \geqslant \frac{1}{\left\|\mathcal{J}\left(x^{*}\right)^{-1}\right\|}\left\|x-x^{*}\right\|-\frac{1}{2 K}\left\|x-x^{*}\right\| \\
& \geqslant\left(\frac{1}{K}-\frac{1}{2 K}\right)\left\|x-x^{*}\right\|=\frac{1}{2 K}\left\|x-x^{*}\right\|,
\end{aligned}
$$

which implies that (3.4) holds.

We state the main result in this section.

Theorem 3.4. Assume there exists $\gamma>0$ such that

$$
\|\nabla f(y)-\nabla f(z)\| \leqslant \gamma\|y-z\| \quad \forall y, z \in N\left(x^{*}, r\right) .
$$

If $x^{(0)} \in N\left(x^{*}, \frac{r}{2}\right)$ satisfies $\mathcal{F}\left(x^{(0)}\right) \neq 0,\left\|\mathcal{F}\left(x^{(0)}\right)\right\| \leqslant \frac{r}{8 K}$, then the MSPIN algorithm generates a sequence $\left\{x^{(k)}\right\} \subset N\left(x^{*}, \frac{r}{2}\right)$ such that $\left\{\left\|\mathcal{F}\left(x^{(k)}\right)\right\|\right\}$ is strictly decreasing, and $x^{(k)} \rightarrow x^{*}$.

Proof. We first prove that the MSPIN algorithm generates a sequence $\left\{x^{(k)}\right\} \subset$ $N\left(x^{*}, \frac{r}{2}\right)$ such that $\left\{\left\|\mathcal{F}\left(x^{(k)}\right)\right\|\right\}$ is strictly decreasing by mathematical induction.

Initial step. In step 2 of Algorithm 2.1, we find $d^{(0)}$ such that

$$
\left\|\mathcal{F}\left(x^{(0)}\right)-\hat{\mathcal{J}}\left(x^{(0)}\right) d^{(0)}\right\| \leq \eta_{0}\left\|\mathcal{F}\left(x^{(0)}\right)\right\|, \quad \eta_{0} \in\left[0, \eta_{\max }\right],
$$

and Lemma 3.2 shows that we could find a suitable step length $\lambda^{(0)}$ and the inequality $\left\|\mathcal{F}\left(x^{(1)}\right)\right\|<\left\|\mathcal{F}\left(x^{(0)}\right)\right\|$ holds, where $x^{(1)}=x^{(0)}-\lambda^{(0)} d^{(0)}, \lambda^{(0)} \in(0,1]$.

From (3.6), we have

$$
\begin{aligned}
\left\|\mathcal{F}\left(x^{(0)}\right)-\hat{\mathcal{J}}\left(x^{(0)}\right)\left(\lambda^{(0)} d^{(0)}\right)\right\| & =\left\|\lambda^{(0)}\left(\mathcal{F}\left(x^{(0)}\right)-\hat{\mathcal{J}}\left(x^{(0)}\right) d^{(0)}\right)+\left(1-\lambda^{(0)}\right) \mathcal{F}\left(x^{(0)}\right)\right\| \\
& \leqslant\left(\eta_{0} \lambda^{(0)}+\left(1-\lambda^{(0)}\right)\right)\left\|\mathcal{F}\left(x^{(0)}\right)\right\| \\
& \leqslant\left\|\mathcal{F}\left(x^{(0)}\right)\right\|,
\end{aligned}
$$

and then it holds from $\left(c_{3}\right)$ in Assumption 3.1 that

$$
\begin{aligned}
\left\|\lambda^{(0)} d^{(0)}\right\| & =\left\|\hat{\mathcal{J}}(x)^{-1}\left(\hat{\mathcal{J}}(x)\left(\lambda^{(0)} d^{(0)}\right)-\mathcal{F}\left(x^{(0)}\right)+\mathcal{F}\left(x^{(0)}\right)\right)\right\| \\
& \leqslant\left\|\hat{\mathcal{J}}(x)^{-1}\right\|\left(\left\|\mathcal{F}\left(x^{(0)}\right)-\hat{\mathcal{J}}\left(x^{(0)}\right)\left(\lambda^{(0)} d^{(0)}\right)\right\|+\left\|\mathcal{F}\left(x^{(0)}\right)\right\|\right) \\
& \leqslant 4 K\left\|\mathcal{F}\left(x^{(0)}\right)\right\| \\
& <4 K \frac{r}{8 K}=\frac{r}{2} .
\end{aligned}
$$

Copyright $\odot$ by SIAM. Unauthorized reproduction of this article is prohibited. 
Hence,

$$
\begin{aligned}
\left\|x^{(1)}-x^{*}\right\| & =\left\|x^{(0)}-x^{*}-\lambda^{(0)} d^{(0)}\right\| \\
& \leqslant\left\|x^{(0)}-x^{*}\right\|+\left\|\lambda^{(0)} d^{(0)}\right\| \\
& <\frac{r}{2}+\frac{r}{2}=r,
\end{aligned}
$$

which implies $x^{(1)} \in N\left(x^{*}, r\right)$. By Lemma 3.3 and $\left\|\mathcal{F}\left(x^{(1)}\right)\right\|<\left\|\mathcal{F}\left(x^{(0)}\right)\right\|$, we have

$$
\left\|x^{(1)}-x^{*}\right\| \leqslant 2 K\left\|\mathcal{F}\left(x^{(1)}\right)\right\|<2 K\left\|\mathcal{F}\left(x^{(0)}\right)\right\|=2 K \frac{r}{8 K}<\frac{r}{2} .
$$

Hence, the MSPIN algorithm can generate $x^{(1)}$ starting from $x^{(0)}$, and $x^{(1)} \in N\left(x^{*}, \frac{r}{2}\right)$.

Inductive step. We assume that there is a $k$ such that the MSPIN algorithm has generated a sequence $\left\{x^{(1)}, x^{(2)}, \ldots, x^{(k)}\right\} \subset N\left(x^{*}, \frac{r}{2}\right)$, and

$$
\left\|\mathcal{F}\left(x^{(k)}\right)\right\|<\cdots<\left\|\mathcal{F}\left(x^{(2)}\right)\right\|<\left\|\mathcal{F}\left(x^{(1)}\right)\right\| .
$$

For a given $x^{(k)}$, we compute the inexact Newton direction $d^{(k)}$ in step 2 of Algorithm 2.1 such that

$$
\left\|\mathcal{F}\left(x^{(k)}\right)-\hat{\mathcal{J}}\left(x^{(k)}\right) d^{(k)}\right\| \leq \eta_{k}\left\|\mathcal{F}\left(x^{(k)}\right)\right\|, \quad \eta_{k} \in\left[0, \eta_{\max }\right] .
$$

Lemma 3.2 guarantees that $x^{(k+1)}=x^{(k)}-\lambda^{(k)} d^{(k)}$ is generated with $\lambda^{(k)} \in(0,1]$ and $\left\|\mathcal{F}\left(x^{(k+1)}\right)\right\|<\left\|\mathcal{F}\left(x^{(k)}\right)\right\|$. Similarly to the procedure in the initial step above, we can prove that $x^{(k+1)} \in N\left(x^{*}, \frac{r}{2}\right)$. This completes the inductive step.

Next we prove that $x^{(k)} \rightarrow x^{*}$. From (3.9), we have

$$
\begin{aligned}
\mathcal{F}\left(x^{(k)}\right)^{T} \hat{\mathcal{J}}\left(x^{(k)}\right) d^{(k)} & =\mathcal{F}\left(x^{(k)}\right)^{T} \mathcal{F}\left(x^{(k)}\right)-\mathcal{F}\left(x^{(k)}\right)^{T}\left(\mathcal{F}\left(x^{(k)}\right)-\hat{\mathcal{J}}\left(x^{(k)}\right) d^{(k)}\right) \\
& \geqslant\left\|\mathcal{F}\left(x^{(k)}\right)\right\|^{2}-\left\|\mathcal{F}\left(x^{(k)}\right)\right\|\left\|\mathcal{F}\left(x^{(k)}\right)-\hat{\mathcal{J}}\left(x^{(k)}\right) d^{(k)}\right\| \\
& \geqslant\left(1-\eta_{k}\right)\left\|\mathcal{F}\left(x^{(k)}\right)\right\|^{2} \\
& \geqslant 2\left(1-\eta_{\max }\right) f\left(x^{(k)}\right),
\end{aligned}
$$

and Lemma 3.2 shows that step 3 of Algorithm 2.1 determines

$$
\lambda^{(k)} \geqslant \min \left\{1, \frac{\alpha \theta_{\min } \mathcal{F}\left(x^{(k)}\right)^{T} \mathcal{J}\left(x^{(k)}\right) d^{(k)}}{\gamma\left\|d^{(k)}\right\|^{2}}\right\}
$$

such that

$$
f\left(x^{(k+1)}\right)=f\left(x^{(k)}-\lambda^{(k)} d^{(k)}\right) \leqslant f\left(x^{(k)}\right)-\alpha \lambda^{(k)} \mathcal{F}\left(x^{(k)}\right)^{T} \hat{\mathcal{J}}\left(x^{(k)}\right) d^{(k)} .
$$

By (3.10) and (3.12), it follows that

$$
f\left(x^{(k+1)}\right) \leqslant\left(1-2 \alpha \lambda^{(k)}\left(1-\eta_{\max }\right)\right) f\left(x^{(k)}\right) .
$$

Note that $\left\{f\left(x^{(k)}\right)\right\}$ is strictly monotonic decreasing and nonnegative, and hence $\lim _{k \rightarrow \infty} f\left(x^{(k)}\right)$ exists and it is nonnegative. We can employ a proof by contradiction to show that $\lim _{k \rightarrow \infty} f\left(x^{(k)}\right)=0$. Assume that

$$
\lim _{k \rightarrow \infty} f\left(x^{(k)}\right)=\sigma>0 .
$$

Copyright $@$ by SIAM. Unauthorized reproduction of this article is prohibited. 
Using (3.9), assumption $\left(c_{2}\right)$, and $\left\|\mathcal{F}\left(x^{(0)}\right)\right\| \leqslant \frac{r}{8 K}$, we have

$$
\begin{aligned}
\left\|d^{(k)}\right\| & =\left\|\hat{\mathcal{J}}(x)^{-1}\left(\hat{\mathcal{J}}(x) d^{(k)}-\mathcal{F}\left(x^{(k)}\right)+\mathcal{F}\left(x^{(k)}\right)\right)\right\| \\
& \leqslant\left\|\hat{\mathcal{J}}(x)^{-1}\right\|\left(\left\|\mathcal{F}\left(x^{(k)}\right)-\hat{\mathcal{J}}\left(x^{(k)}\right) d^{(k)}\right\|+\left\|\mathcal{F}\left(x^{(k)}\right)\right\|\right) \\
& \leqslant 2 K\left(1+\eta_{k}\right)\left\|\mathcal{F}\left(x^{(k)}\right)\right\| \\
& \leqslant 2 K\left(1+\eta_{\max }\right)\left\|\mathcal{F}\left(x^{(0)}\right)\right\| \\
& <2 K\left(1+\eta_{\max }\right) \frac{r}{8 K}=\frac{r\left(1+\eta_{\max }\right)}{4},
\end{aligned}
$$

and then it follows from (3.10) that

$$
\frac{\mathcal{F}\left(x^{(k)}\right)^{T} \mathcal{J}\left(x^{(k)}\right) d^{(k)}}{\left\|d^{(k)}\right\|^{2}}>\frac{2\left(1-\eta_{\max }\right) f\left(x^{(k)}\right)}{\left(\frac{r\left(1+\eta_{\max }\right)}{4}\right)^{2}} \geqslant \frac{32\left(1-\eta_{\max }\right) \sigma}{r^{2}\left(1+\eta_{\max }\right)^{2}}>0 .
$$

Let $\epsilon=\frac{1}{2} \min \left\{1, \frac{32 \alpha \theta_{\min }\left(1-\eta_{\max }\right) \sigma}{\gamma r^{2}\left(1+\eta_{\max }\right)^{2}}\right\} \leqslant \frac{1}{2}$. Therefore, by (3.11) and (3.16), we have

$$
\lambda^{(k)}>\min \left\{1, \frac{32 \alpha \theta_{\min }\left(1-\eta_{\max }\right) \sigma}{\gamma r^{2}\left(1+\eta_{\max }\right)^{2}}\right\}>\epsilon>0,
$$

which implies that

$$
\lim _{k \rightarrow \infty} \frac{f\left(x^{(k+1)}\right)}{f\left(x^{(k)}\right)} \leqslant 1-2 \alpha \epsilon\left(1-\eta_{\max }\right)<1
$$

holds for $\alpha \in\left(0, \frac{1}{2}\right)$ and $\eta_{\max } \in(0,1)$ (given in Algorithm 2.1) from (3.13). It is then seen from (3.14) that

$$
\lim _{k \rightarrow \infty} \frac{f\left(x^{(k+1)}\right)}{f\left(x^{(k)}\right)}=1,
$$

which contradicts (3.18). Thus, we have $\lim _{k \rightarrow \infty} f\left(x^{(k)}\right)=0$, and then Lemma 3.3 implies that

$$
\left\|x^{(k)}-x^{*}\right\| \leqslant 2 K\left\|\mathcal{F}\left(x^{(k)}\right)\right\|=2 K \sqrt{2 f\left(x^{(k)}\right)} \rightarrow 0,
$$

i.e., $x^{(k)} \rightarrow x^{*}$ as $k \rightarrow \infty$.

4. Convergence rate. We discuss the local convergence of the MSPIN algorithm in the previous section. In this section, we investigate the convergence rate of the MSPIN algorithm under some reasonable assumptions. For reference, here as Lemma 4.1 we reproduce Theorem 4.1 from [1].

Lemma 4.1. Let $f(x)$ be twice continuously differentiable in $N\left(x^{*}, r\right)$ and there exists $\beta>0$ such that

$$
\|\nabla f(y)-\nabla f(z)\| \leqslant \beta\|y-z\|, \quad\left\|\nabla^{2} f(y)-\nabla^{2} f(z)\right\| \leqslant \beta\|y-z\|
$$

hold for any $y, z \in N\left(x^{*}, r\right)$. Let $\left\{x^{(k)}\right\}$ be the sequence generated by the MSPIN method such that $x^{(k)} \rightarrow x^{*}$. If $\eta_{k} \rightarrow 0$, then $x^{(k+1)}=x^{(k)}-d^{(k)}$ for all sufficiently large $k$. 
Lemma 4.1 implies that under reasonable conditions on $\nabla f(x)$ and $\nabla^{2} f(x)$, the while-loop in step 3 of Algorithm 2.1 is not executed and $\lambda^{(k)}=1$ is acceptable when $k$ is sufficiently large.

Under the conditions of (c) in Assumption 3.1, the following lemma shows that $\mathcal{J}(x)$ and $\hat{\mathcal{J}}(x)$ are Lipschitz continuous in a neighborhood of $x^{*}$.

Lemma 4.2. Let $F(x)$ be twice continuously differentiable in $N\left(x^{*}, r\right)$, and $\mathcal{J}(x)$ and $\hat{\mathcal{J}}(x)$ are defined in (2.29) and (2.35), respectively. Then there is a neighborhood $V \subset N\left(x^{*}, \frac{r}{2}\right)$ of the exact solution $x^{*}$ such that both $\mathcal{J}(x)$ and $\hat{\mathcal{J}}(x)$ are Lipschitz continuous in $V$.

Proof. In the neighborhood $N\left(x^{*}, \frac{r}{2}\right), F^{\prime \prime}(x), T_{i}^{\prime}(x), L(x)^{-1}$ and $E_{i} J(x)$ are continuous, and then we define

$$
\begin{aligned}
M_{1} & :=\sup _{x \in N\left(x^{*}, \frac{r}{2}\right)}\left\|F^{\prime \prime}(x)\right\|, \quad M_{2}:=\max _{i} \sup _{x \in N\left(x^{*}, \frac{r}{2}\right)}\left\|\tilde{T}_{i}^{\prime}(x)\right\|, \\
M_{3} & :=\sup _{x \in N\left(x^{*}, \frac{r}{2}\right)}\left\|L(x)^{-1}\right\|, \quad M_{4}:=\max _{i} \sup _{x \in N\left(x^{*}, \frac{r}{2}\right)}\left\|E_{i} J(x)\right\|,
\end{aligned}
$$

where $E_{i}$ is defined in (2.7), $\tilde{T}_{i} \in R^{n}$ is defined in (2.14), and $\tilde{T}_{i}^{\prime}(x)=\sum_{k=1}^{i} R_{k}^{T} T_{k}^{\prime}(x)$.

For any $x, y \in N\left(x^{*}, \frac{r}{2}\right)$, by Lemma 3.3.5 and Lemma 3.2.3 in [15], we have

$$
\begin{aligned}
& \|J(x)-J(y)\| \leqslant \sup _{0 \leqslant t \leqslant 1} \| F^{\prime \prime}\left(x+t(y-x)\|\| x-y\left\|\leqslant M_{1}\right\| x-y \|,\right. \\
& \left\|\tilde{T}_{i}(x)-\tilde{T}_{i}(y)\right\| \leqslant \sup _{0 \leqslant t \leqslant 1} \| \tilde{T}_{i}^{\prime}\left(x+t(y-x)\|\| x-y\left\|\leqslant M_{2}\right\| x-y \|,\right.
\end{aligned}
$$

where $i=1,2, \ldots, N$. By (4.1) and since $L(x)$ is a lower triangular matrix, we have

$$
\|L(x)-L(y)\| \leqslant \max _{i}\left\|E_{i}(J(x)-J(y)) E_{i}\right\| \leqslant\|J(x)-J(y)\| \leqslant M_{1}\|x-y\| .
$$

Since $T_{i}(x)$ is continuous and $\tilde{T}_{i}(x)=\sum_{k=1}^{i} R_{k}^{T} T_{k}(x)$, then $x-\tilde{T}_{i}(x) \rightarrow x^{*}$ as $x \rightarrow x^{*}$, and hence there exists a neighborhood $V_{i} \subset N\left(x^{*}, \frac{r}{2}\right)$ such that $x-\tilde{T}_{i}(x) \in N\left(x^{*}, \frac{r}{2}\right)$ for any $x \in V_{i}, i=1,2 \ldots, N$. We set $V=\bigcap_{i=1}^{N} V_{i} \subset N\left(x^{*}, \frac{r}{2}\right)$. For any $x, y \in V$ and for each $i$, by (4.1) and (4.2), it follows that

$$
\begin{aligned}
\left\|J\left(x-\tilde{T}_{i}(x)\right)-J\left(y-\tilde{T}_{i}(y)\right)\right\| & \leqslant M_{1}\left\|(x-y)-\left(\tilde{T}_{i}(x)-\tilde{T}_{i}(y)\right)\right\| \\
& \leqslant M_{1}\left(1+M_{2}\right)\|x-y\| .
\end{aligned}
$$

In a similar way, we have

$$
\left\|L\left(x-\tilde{T}_{i}(x)\right)-L\left(y-\tilde{T}_{i}(y)\right)\right\| \leqslant M_{1}\left(1+M_{2}\right)\|x-y\|,
$$

where $i=1,2, \ldots, N$.

By setting

$$
\hat{L}(x)=L\left(x-\tilde{T}_{N}(x)\right), \quad B_{i}(x)=E_{i} J\left(x-\tilde{T}_{i}(x)\right), \quad i=1,2, \ldots, N,
$$

we recast $(2.29)$ as

$$
\mathcal{J}(x)=L\left(x-\tilde{T}_{N}(x)\right)^{-1} \sum_{i=1}^{N} E_{i} J\left(x-\tilde{T}_{i}(x)\right)=\sum_{i=1}^{N} \mathcal{J}_{i}(x),
$$

Copyright $@$ by SIAM. Unauthorized reproduction of this article is prohibited. 
where $\mathcal{J}_{i}(x)=\hat{L}(x)^{-1} B_{i}(x)$. Using (4.4) and (4.5), for any $x, y \in V$, we have

$$
\begin{aligned}
\left\|\mathcal{J}_{i}(x)-\mathcal{J}_{i}(y)\right\|= & \left\|\hat{L}(x)^{-1} B_{i}(x)-\hat{L}(y)^{-1} B_{i}(y)\right\| \\
\leqslant & \left\|\hat{L}(x)^{-1} B_{i}(x)-\hat{L}(x)^{-1} B_{i}(y)\right\| \\
& +\left\|\hat{L}(x)^{-1} B_{i}(y)-\hat{L}(y)^{-1} B_{i}(y)\right\| \\
\leqslant & \left\|\hat{L}(x)^{-1}\right\|\left\|B_{i}(x)-B_{i}(y)\right\| \\
& +\left\|\hat{L}(x)^{-1}\right\|\|\hat{L}(x)-\hat{L}(y)\|\left\|\hat{L}(y)^{-1}\right\|\left\|B_{i}(y)\right\| \\
\leqslant & M_{3} M_{1}\left(1+M_{2}\right)\|x-y\|+M_{3}^{2} M_{1}\left(1+M_{2}\right) M_{4}\|x-y\| \\
= & M_{1} M_{3}\left(1+M_{2}\right)\left(1+M_{3} M_{4}\right)\|x-y\| .
\end{aligned}
$$

Thus,

$$
\begin{aligned}
\|\mathcal{J}(x)-\mathcal{J}(y)\| & =\left\|\sum_{i=1}^{N} \mathcal{J}_{i}(x)-\sum_{i=1}^{N} \mathcal{J}_{i}(y)\right\| \leqslant \sum_{i=1}^{N}\left\|\mathcal{J}_{i}(x)-\mathcal{J}_{i}(y)\right\| \\
& \leqslant N M_{1} M_{3}\left(1+M_{2}\right)\left(1+M_{3} M_{4}\right)\|x-y\|
\end{aligned}
$$

which shows that $\mathcal{J}(x)$ is Lipschitz continuous in $V$.

We recast $(2.35)$ as

$$
\hat{\mathcal{J}}(x)=L\left(x-\tilde{T}_{N-1}(x)\right)^{-1} J\left(x-\tilde{T}_{N-1}(x)\right)=\sum_{i=1}^{N} \hat{\mathcal{J}}_{i}(x),
$$

where $\hat{\mathcal{J}}_{i}(x)=L\left(x-\tilde{T}_{N-1}(x)\right)^{-1} E_{i} J\left(x-\tilde{T}_{N-1}(x)\right)$. Similarly to (4.8), we can prove that

$$
\|\hat{\mathcal{J}}(x)-\hat{\mathcal{J}}(y)\| \leqslant N M_{1} M_{3}\left(1+M_{2}\right)\left(1+M_{3} M_{4}\right)\|x-y\|
$$

for any $x, y \in V$. Therefore, $\hat{\mathcal{J}}(x)$ is also Lipschitz continuous in $V$.

TheOREM 4.3. Let $F(x)$ and $f(x)$ be twice continuously differentiable in $N\left(x^{*}, r\right)$, and there exists a constant $C>0$ such that

$$
\|\nabla f(x)-\nabla f(y)\| \leqslant C\|x-y\|, \quad\left\|\nabla^{2} f(x)-\nabla^{2} f(y)\right\| \leqslant C\|x-y\|
$$

for any $x, y \in N\left(x^{*}, r\right)$. Consider a sequence $\left\{x^{(k)}\right\}$ generated by the MSPIN method such that $x^{(k)} \rightarrow x^{*}$, and then

(i) if $\eta_{k} \rightarrow 0, x^{(k)} \rightarrow x^{*}$ superlinearly;

(ii) if $\eta_{k}=\mathcal{O}\left(\left\|\mathcal{F}\left(x^{(k)}\right)\right\|\right), x^{(k)} \rightarrow x^{*}$ quadratically.

Proof. According to Lemma 4.1, we have

$$
x^{(k+1)}=x^{(k)}-d^{(k)},
$$

where $\left\|\mathcal{F}\left(x^{(k)}\right)-\hat{\mathcal{J}}\left(x^{(k)}\right) d^{(k)}\right\| \leq \eta_{k}\left\|\mathcal{F}\left(x^{(k)}\right)\right\|$, if $\eta_{k} \rightarrow 0$ for all the sufficiently large $k$. By Lemma 4.2, there exists a $\gamma>0$ and a neighborhood $V \subset N\left(x^{*}, \frac{r}{2}\right)$ such that $\mathcal{J}(x)$ and $\hat{\mathcal{J}}(x)$ are Lipschitz continuous with Lipschitz constant $\gamma$ in $V$.

Let $k$ be large enough such that $x^{(k)}, x^{*}+t\left(x^{(k)}-x^{*}\right) \in V$ and (4.13) hold. Then we obtain

$$
\begin{aligned}
& \left\|\hat{\mathcal{J}}\left(x^{(k)}\right)-\mathcal{J}\left(x^{*}+t\left(x^{(k)}-x^{*}\right)\right)\right\| \\
& \quad \leqslant\left\|\hat{\mathcal{J}}\left(x^{(k)}\right)-\hat{\mathcal{J}}\left(x^{*}\right)\right\|+\left\|\mathcal{J}\left(x^{*}\right)-\mathcal{J}\left(x^{*}+t\left(x^{(k)}-x^{*}\right)\right)\right\| \\
& \quad \leqslant \gamma(1+t)\left\|x^{(k)}-x^{*}\right\|, \quad t \in[0,1] .
\end{aligned}
$$

Copyright $\odot$ by SIAM. Unauthorized reproduction of this article is prohibited. 
The mean value theorem shows that

$$
\mathcal{F}\left(x^{(k)}\right)=\mathcal{F}\left(x^{(k)}\right)-\mathcal{F}\left(x^{*}\right)=\left(\int_{0}^{1} \mathcal{J}\left(x^{*}+t\left(x^{(k)}-x^{*}\right)\right) d t\right)\left(x^{(k)}-x^{*}\right) .
$$

Note that $\left\|\hat{\mathcal{J}}\left(x^{(k)}\right)^{-1}\right\| \leqslant 2 K$ because $x^{(k)} \in N\left(x^{*}, r\right)$. Using (4.14) and (4.15),

$$
\begin{aligned}
& \left\|x^{(k)}-x^{*}-\hat{\mathcal{J}}\left(x^{(k)}\right)^{-1} \mathcal{F}\left(x^{(k)}\right)\right\| \\
& \quad \leqslant\left\|\hat{\mathcal{J}}\left(x^{(k)}\right)^{-1}\right\|\left(\int_{0}^{1}\left\|\hat{\mathcal{J}}\left(x^{(k)}\right)-\mathcal{J}\left(x^{*}+t\left(x^{(k)}-x^{*}\right)\right)\right\| d t\right)\left\|\left(x^{(k)}-x^{*}\right)\right\| \\
& \quad \leqslant 3 \gamma K\left\|\left(x^{(k)}-x^{*}\right)\right\|^{2} .
\end{aligned}
$$

From (4.15), it holds from $\left(\mathrm{c}_{2}\right)$ in Assumption 3.1 that

$$
\left\|\mathcal{F}\left(x^{(k)}\right)\right\| \leqslant\left(\int_{0}^{1}\left\|\mathcal{J}\left(x^{*}+t\left(x^{(k)}-x^{*}\right) \| d t\right)\right\| x^{(k)}-x^{*}\|\leqslant 2 K\| x^{(k)}-x^{*} \|,\right.
$$

and it follows that

$$
\begin{aligned}
\left\|\hat{\mathcal{J}}\left(x^{(k)}\right)^{-1} \mathcal{F}\left(x^{(k)}\right)-d^{(k)}\right\| & \leqslant\left\|\hat{\mathcal{J}}\left(x^{(k)}\right)^{-1}\right\|\left\|\mathcal{F}\left(x^{(k)}\right)-\hat{\mathcal{J}}\left(x^{(k)}\right) d^{(k)}\right\| \\
& \leqslant \eta_{k}\left\|\hat{\mathcal{J}}\left(x^{(k)}\right)^{-1}\right\|\left\|\mathcal{F}\left(x^{(k)}\right)\right\| \\
& \leqslant 4 \eta_{k} K^{2}\left\|x^{(k)}-x^{*}\right\| .
\end{aligned}
$$

Finally, we note that

$$
\begin{aligned}
x^{(k+1)}-x^{*} & =\left(x^{(k)}-x^{*}\right)+\left(x^{(k+1)}-x^{(k)}\right) \\
& =\left(x^{(k)}-x^{*}\right)-d^{(k)} \\
& =\left(x^{(k)}-x^{*}-\hat{\mathcal{J}}\left(x^{(k)}\right)^{-1} \mathcal{F}\left(x^{(k)}\right)\right)+\left(\hat{\mathcal{J}}\left(x^{(k)}\right)^{-1} \mathcal{F}\left(x^{(k)}\right)-d^{(k)}\right),
\end{aligned}
$$

and then we use (4.16) and (4.18) to conclude

$$
\left\|x^{(k+1)}-x^{*}\right\| \leqslant 3 \gamma K\left\|x^{(k)}-x^{*}\right\|^{2}+4 \eta_{k} K^{2}\left\|x^{(k)}-x^{*}\right\|,
$$

which completes the proof.

Theorem 4.3 implies that the choice of the forcing term $\eta_{k}$ influences the convergence rate of the MSPIN algorithm. The Newton iteration can achieve superlinear or even quadratic rates of convergence by appropriately choosing $\eta_{k}$.

5. Conclusion. The MSPIN algorithm [13] was introduced as a physics-based field-split method to cope with "nonlinear stiffness"; however, until now the convergence of MSPIN has not been discussed and no theory predicts even local convergence rates.

In this paper, we provide the MSPIN algorithm with a local convergence proof. Based on local assumptions on $F(x)$, it is shown that the preconditioned function $\mathcal{F}(x)$ is continuously differentiable, and we obtain the Jacobian of the transformed system $\mathcal{F}^{\prime}(x)$ in a more intuitive way than previously presented. Under some reasonable assumptions, we discuss the local convergence property and the convergence rate. Superlinear or even quadratic convergence can be obtained when the forcing terms $\eta_{k}$ are picked suitably.

Appendix A. An example. In this section, a simple example illustrates that the conditions $\left(c_{1}\right)-\left(c_{5}\right)$ in Assumption 3.1 are not unreasonably strict. The following lemma is required in the later discussion. 
Lemma A.1.

$$
\begin{aligned}
& 1+x<e^{x}<1+3 x, \quad x \in(0,1), \\
& e^{x} \leqslant 1+\frac{1}{2} x, \quad x \in(-1,0], \\
& \left|e^{x}-1\right| \leqslant e^{|x|}-1 .
\end{aligned}
$$

Consider the system $F(x)$ of two nonlinear equations in two unknowns,

$$
\begin{aligned}
& F_{1}\left(x_{1}, x_{2}\right)=\left(x_{1}-x_{2}^{3}-1\right)^{3}-x_{2}^{3}, \\
& F_{2}\left(x_{1}, x_{2}\right)=x_{1}+2 \mu e^{x_{2}}-1-2 \mu,
\end{aligned}
$$

where $\mu \in(0,1)$. It is easy to verify that $x^{*}=[1,0]^{T}$ is a solution of this system. The nonlinearly preconditioned system $\mathcal{F}(x)=\left[T_{1}(x), T_{2}(x)\right]^{T}$ is obtained by solving

$$
\begin{aligned}
F_{1}\left(x_{1}-T_{1}(x), x_{2}\right) & =\left(x_{1}-T_{1}(x)-x_{2}^{3}-1\right)^{3}-x_{2}^{3}=0, \\
F_{2}\left(x_{1}-T_{1}(x), x_{2}-T_{2}\right) & =x_{1}-T_{1}(x)+2 \mu e^{x_{2}-T_{2}(x)}-1-2 \mu=0,
\end{aligned}
$$

from which we get

$$
x_{1}-T_{1}(x)-x_{2}^{3}-1=x_{2},
$$

and it follows that

$$
F_{2}\left(x_{1}-T_{1}(x), x_{2}-T_{2}(x)\right)=x_{2}^{3}+x_{2}-2 \mu+2 \mu e^{x_{2}-T_{2}(x)}=0 .
$$

Thus, we obtain

$$
\mathcal{F}^{\prime}(x)=\mathcal{J}(x)=\left[\begin{array}{cc}
1 & -3 x_{2}^{2}-1 \\
0 & 1+\frac{1}{2 \mu}\left(3 x_{2}^{2}+1\right) e^{-\left(x_{2}-T_{2}(x)\right)}
\end{array}\right]
$$

and

$$
\hat{\mathcal{J}}(x)=\left[\begin{array}{cc}
1 & -3 x_{2}^{2}-1 \\
0 & 1+\frac{1}{2 \mu}\left(3 x_{2}^{2}+1\right) e^{-x_{2}}
\end{array}\right] .
$$

Moreover,

$$
\mathcal{J}\left(x^{*}\right)=\hat{\mathcal{J}}\left(x^{*}\right)=\left[\begin{array}{cc}
1 & -1 \\
0 & 1+\frac{1}{2 \mu}
\end{array}\right]
$$

and

$$
K=\max \left\{\left\|\mathcal{J}\left(x^{*}\right)\right\|,\left\|\mathcal{J}\left(x^{*}\right)^{-1}\right\|\right\}=1+\frac{1}{2 \mu} .
$$

Besides, the continuity of $L(x)^{-1}, \mathcal{J}(x), \hat{\mathcal{J}}(x), \mathcal{J}(x)^{-1}$, and $\hat{\mathcal{J}}(x)^{-1}$ is easily verified. Let $r \in\left(0, \frac{\mu}{3}\right)$ and we assume that $x=\left[x_{1}, x_{2}\right]^{T} \in N\left(x^{*}, r\right)$, i.e.,

$$
\left(x_{1}-1\right)^{2}+x_{2}^{2} \leqslant r^{2},
$$

Copyright $@$ by SIAM. Unauthorized reproduction of this article is prohibited. 
which implies that $\left|x_{2}\right| \leqslant r$. Using (A.1) and (A.5), it holds that

$$
\begin{aligned}
& e^{-x_{2}} \leqslant e^{\left|x_{2}\right|} \leqslant e^{r}<e^{\frac{\mu}{3}}<1+\mu<2, \\
& 3 x_{2}^{2}+1 \leqslant 3 r^{2}+1<3\left(\frac{\mu}{3}\right)^{2}+1<\frac{1}{3} \mu^{2}+1, \\
& \frac{1}{2 \mu} e^{-\left(x_{2}-T_{2}(x)\right)}=\frac{1}{2 \mu-x_{2}^{3}-x_{2}}<\frac{1}{2 \mu-r^{3}-r}<\frac{1}{\mu+(\mu-2 r)}<\frac{1}{\mu},
\end{aligned}
$$

and it follows that
(A.10)
$\|\hat{\mathcal{J}}(x)\|=1+\frac{1}{2 \mu}\left(3 x_{2}^{2}+1\right) e^{-x_{2}} \leqslant 1+\frac{1}{\mu}\left(\frac{1}{3} \mu^{2}+1\right)<2+\frac{1}{\mu}=2 K$,
(A.11)
$\|\mathcal{J}(x)\|=1+\frac{1}{2 \mu}\left(3 x_{2}^{2}+1\right) e^{-\left(x_{2}-T_{2}(x)\right)} \leqslant 1+\frac{1}{\mu}\left(\frac{1}{3} \mu^{2}+1\right)<2+\frac{1}{\mu}=2 K$.

Note that

$$
\left\|\mathcal{J}(x)^{-1}\right\|=1 \leqslant 2 K, \quad\left\|\hat{\mathcal{J}}(x)^{-1}\right\|=1 \leqslant 2 K \quad \forall x \in N\left(x^{*}, r\right) .
$$

Since

$$
\left|\frac{1}{2 \mu}\left(x_{2}^{3}+x_{2}\right)\right| \leqslant \frac{1}{2 \mu}\left(r^{3}+r\right) \leqslant \frac{r}{\mu}<\frac{1}{3}, \quad r \in\left(0, \frac{\mu}{3}\right),
$$

by (A.5), it holds that

$$
e^{-1}<1-\frac{1}{3}<e^{x_{2}-T_{2}(x)}=1-\frac{1}{2 \mu}\left(x_{2}^{3}+x_{2}\right)<1+\frac{1}{3}<e,
$$

which implies that $\left|x_{2}-T_{2}(x)\right|<1$. From (A.1), (A.2), and (A.14), we have

$$
\begin{gathered}
x_{2}-T_{2}(x)<-\frac{1}{2 \mu}\left(x_{2}^{3}+x_{2}\right) \leqslant \frac{1}{2 \mu}\left(\left|x_{2}\right|^{3}+\left|x_{2}\right|\right) \quad \text { if } x_{2}-T_{2}(x)>0, \\
-\frac{1}{\mu}\left(\left|x_{2}\right|^{3}+\left|x_{2}\right|\right) \leqslant-\frac{1}{\mu}\left(x_{2}^{3}+x_{2}\right) \leqslant x_{2}-T_{2} \quad \text { if } x_{2}-T_{2}(x) \leqslant 0 .
\end{gathered}
$$

Hence,

$$
\left|x_{2}-T_{2}(x)\right| \leqslant \frac{1}{\mu}\left(\left|x_{2}\right|^{2}+1\right)\left|x_{2}\right| \leqslant \frac{1}{\mu}(r+1)\left|x_{2}\right|<\left(\frac{1}{3}+\frac{1}{\mu}\right)\left|x_{2}\right|,
$$

and then we have

$$
\left|T_{2}(x)\right| \leqslant\left|x_{2}-T_{2}(x)\right|+\left|x_{2}\right|<\left(\frac{4}{3}+\frac{1}{\mu}\right) r<\frac{4}{9} \mu+\frac{1}{3}<1, \quad r \in\left(0, \frac{\mu}{3}\right) .
$$

According to (A.1), (A.3), and (A.17), we have

$$
\begin{aligned}
\|\mathcal{J}(x)-\hat{\mathcal{J}}(x)\| & =\frac{1}{2 \mu}\left(3 x_{2}^{2}+1\right) e^{-x_{2}}\left|e^{T_{2}(x)}-1\right| \\
& \leqslant \frac{1}{2 \mu}\left(\frac{1}{3} \mu^{2}+1\right) e^{r}\left(e^{\left|T_{2}(x)\right|}-1\right) \\
& \leqslant \frac{1}{2 \mu}\left(\frac{1}{3}+1\right) e^{r}\left(3\left|T_{2}(x)\right|\right) \\
& \leqslant \frac{2 e^{r}}{3 \mu}\left[3\left(\frac{4}{3}+\frac{1}{\mu}\right) r\right] \leqslant \frac{2 e r}{\mu}\left(\frac{4}{3}+\frac{1}{\mu}\right) .
\end{aligned}
$$

Copyright $@$ by SIAM. Unauthorized reproduction of this article is prohibited. 
It is seen that condition $\left(c_{4}\right)$ is satisfied when

$$
r \leqslant \frac{\mu\left(1-\eta_{\max }\right)}{8 e K\left(\frac{4}{3}+\frac{1}{\mu}\right)\left(1+\eta_{\max }\right)}=\frac{\mu\left(1-\eta_{\max }\right)}{4 e\left(2+\frac{1}{\mu}\right)\left(\frac{4}{3}+\frac{1}{\mu}\right)\left(1+\eta_{\max }\right)},
$$

and then it is noted that

$$
\frac{\mu\left(1-\eta_{\max }\right)}{8 e K\left(\frac{4}{3}+\frac{1}{\mu}\right)\left(1+\eta_{\max }\right)} \leqslant \frac{\mu}{8 e K\left(\frac{4}{3}+\frac{1}{\mu}\right)}=\frac{3 \mu^{2}}{8 e K(4 \mu+3)} \leqslant \frac{\mu^{2}}{8 e K}<\frac{\mu^{2}}{3 K}<\frac{\mu}{3} .
$$

From (A.5) and (A.6), we have

$$
\mathcal{J}(x)=\left[\begin{array}{cc}
1 & -3 x_{2}^{2}-1 \\
0 & 1+\frac{1}{2 \mu} \frac{3 x_{2}^{2}+1}{1-\frac{1}{2 \mu}\left(x_{2}^{3}+x_{2}\right)}
\end{array}\right],
$$

and it follows that

$$
\left\|\mathcal{J}\left(x^{*}+t\left(x-x^{*}\right)\right)-\mathcal{J}\left(x^{*}\right)\right\|=\frac{1}{2 \mu}\left|\frac{3\left(t x_{2}\right)^{2}+1}{1-\frac{1}{2 \mu}\left(\left(t x_{2}\right)^{3}+\left(t x_{2}\right)\right)}-1\right|, t \in[0,1] .
$$

Note that $K=1+\frac{1}{2 \mu}>1, \mu \in(0,1)$, and $\left|x_{2}\right|<r$. Letting $0<r \leqslant \frac{\mu^{2}}{3 K}<\frac{\mu}{3}<1$, we have

$$
\left|\frac{1}{2 \mu}\left(\left(t x_{2}\right)^{3}+\left(t x_{2}\right)\right)\right| \leqslant \frac{1}{2 \mu}\left(\left|x_{2}\right|^{3}+\left|x_{2}\right|\right) \leqslant \frac{1}{2 \mu}\left(r^{3}+r\right) \leqslant \frac{r}{\mu} \leqslant \frac{\mu}{3 K}<1,
$$

and then it holds that

$$
\begin{gathered}
-\frac{\mu}{\mu+3 K}=\frac{1}{1+\frac{\mu}{3 K}}-1 \leqslant \frac{3\left(t x_{2}\right)^{2}+1}{1-\frac{1}{2 \mu}\left(\left(t x_{2}\right)^{3}+\left(t x_{2}\right)\right)}-1, \\
\frac{3\left(t x_{2}\right)^{2}+1}{1-\frac{1}{2 \mu}\left(\left(t x_{2}\right)^{3}+\left(t x_{2}\right)\right)}-1 \leqslant \frac{3 r^{2}+1}{1-\frac{\mu}{3 K}}-1 \leqslant \frac{\mu r+1}{1-\frac{\mu}{3 K}}-1 .
\end{gathered}
$$

From (A.21), (A.23), and (A.24), we have

$$
\sup _{0 \leqslant t \leqslant 1}\left\|\mathcal{J}\left(x^{*}+t\left(x-x^{*}\right)\right)-\mathcal{J}\left(x^{*}\right)\right\| \leqslant \max \left\{\frac{1}{2(\mu+3 K)}, \frac{1}{2 \mu}\left(\frac{\mu r+1}{1-\frac{\mu}{3 K}}-1\right)\right\} .
$$

According to the mean value theorem, we have

$$
\begin{aligned}
& \left\|\mathcal{F}(x)-\mathcal{F}\left(x^{*}\right)-\mathcal{J}\left(x^{*}\right)\left(x-x^{*}\right)\right\| \\
& \quad=\left\|\left(\int_{0}^{1}\left(\mathcal{J}\left(x^{*}+t\left(x-x^{*}\right)\right)-\mathcal{J}\left(x^{*}\right)\right) d t\right)\left(x-x^{*}\right)\right\| \\
& \quad \leqslant \sup _{0 \leqslant t \leqslant 1}\left\|\mathcal{J}\left(x^{*}+t\left(x-x^{*}\right)\right)-\mathcal{J}\left(x^{*}\right)\right\|\left\|x-x^{*}\right\| .
\end{aligned}
$$

Therefore, it is seen that condition $\left(c_{5}\right)$ is satisfied when

$$
\frac{1}{2 \mu}\left(\frac{\mu r+1}{1-\frac{\mu}{3 K}}-1\right) \leqslant \frac{1}{2 K}
$$

Copyright (c) by SIAM. Unauthorized reproduction of this article is prohibited. 
from which we obtain

$$
r \leqslant \frac{1}{3 K}\left(2-\frac{\mu}{K}\right)
$$

It is easily verified that

$$
\frac{\mu^{2}}{3 K} \leqslant \frac{1}{3 K}\left(2-\frac{\mu}{K}\right)
$$

because $\mu^{2}+\frac{\mu}{K}=\mu^{2}+\frac{\mu}{1+\frac{1}{2 \mu}} \leqslant 2 \mu \leqslant 2$.

Using (A.18), (A.19), (A.27), and (A.28), to sum up, assumptions $\left(\mathrm{c}_{1}\right)-\left(\mathrm{c}_{5}\right)$ hold for any $x \in N\left(x^{*}, r\right)$, where $r$ satisfies the following inequality:

$$
r \leqslant \frac{\mu\left(1-\eta_{\max }\right)}{4 e\left(2+\frac{1}{\mu}\right)\left(\frac{4}{3}+\frac{1}{\mu}\right)\left(1+\eta_{\max }\right)} .
$$

Acknowledgment. The authors thank anonymous referees for excellent suggestions on improving the paper.

\section{REFERENCES}

[1] H.-B. An, On convergence of the additive Schwarz preconditioned inexact Newton method, SIAM J. Numer. Anal., 43 (2005), pp. 1850-1871.

[2] X.-C. Cai and D. E. Keyes, Nonlinearly preconditioned inexact Newton algorithms, SIAM J. Sci. Comput., 24 (2002), pp. 183-200.

[3] X.-C. Cai, D. E. Keyes, and L. Marcinkowski, Nonlinear additive Schwarz preconditioners and application in computational fluid dynamics, Internat. J. Numer. Methods Fluids, 40 (2002), pp. 1463-1470.

[4] X.-C. Cai, D. E. Keyes, And D. P. Young, A nonlinear additive Schwarz preconditioned inexact Newton method for shocked duct flow, in Proceedings of the 13th International Conference on Domain Decomposition Methods, Lyon, France, 2000, pp. 343-350.

[5] J. E. Dennis and R. B. Schnabel, Numerical Methods for Unconstrained Optimization and Nonlinear Equations, SIAM, Philadelphia, 1996.

[6] M. Dryja And W. HackBusch, On the nonlinear domain decomposition method, BIT, 37 (1997), pp. 296-311.

[7] S. C. Eisenstat and H. F. Walker, Globally convergent inexact Newton methods, SIAM J. Optim., 4 (1994), pp. 393-422.

[8] S. C. Eisenstat and H. F. Walker, Choosing the forcing terms in an inexact Newton method, SIAM J. Sci. Comput., 17 (1996), pp. 16-32.

[9] R. Ernst, B. Flemisch, And B. Wohlmuth, A multiplicative Schwarz method and its application to nonlinear acoustic-structure interaction, ESAIM Math. Model. Numer. Anal., 43 (2009), pp. 487-506.

[10] F.-N. Hwang AND X.-C. CAI, A parallel nonlinear additive Schwarz preconditioned inexact Newton algorithm for incompressible Navier-Stokes equations, J. Comput. Phys., 204 (2005), pp. 666-691.

[11] C. T. KeLLEY, Iterative Methods for Linear and Nonlinear Equations, SIAM, Philadelphia, 1995.

[12] D. A. Knoll and D. E. Keyes, Jacobian-free Newton-Krylov methods: A survey of approaches and applications, J. Comput. Phys., 193 (2004), pp. 357-397.

[13] L. Liu And D. E. Keyes, Field-split preconditioned inexact Newton algorithms, SIAM J. Sci. Comput., 37 (2015), pp. 1388-1409.

[14] S. H. LuI, Nonlinearly preconditioned Newton's method, in Proceedings of the 14th International Conference on Domain Decomposition Methods, Cocoyoc, Mexico, 2002, pp. 95-105.

[15] J. M. Ortega And W. C. Rheinboldt, Iterative Solution of Nonlinear Equations in Several Variables, SIAM, Philadelphia, 2000.

[16] M. Pernice And H. F. WALker, NITSOL: A Newton iterative solver for nonlinear systems, SIAM J. Sci. Comput., 19 (1998), pp. 302-318.

[17] Y. SAAD, Iterative Methods for Sparse Linear Systems, 2nd ed., SIAM, Philadelphia, 2003.

Copyright (C) by SIAM. Unauthorized reproduction of this article is prohibited. 
[18] Y. SaAd And M. H. Schultz, GMRES: A generalized minimal residual algorithm for solving nonsymmetric linear systems, SIAM J. Sci. Statist. Comput., 7 (1986), pp. 856-869.

[19] J. O. Skogestad, E. Keilegavlen, And J. M. Nordbotten, Domain decomposition strategies for nonlinear flow problems in porous media, J. Comput. Phys., (2013), pp. 439-451.

[20] J. O. Skogestad, E. Keilegavlen, and J. M. Nordbotten, Two-scale preconditioning for two-phase nonlinear flows in porous media, Transp. Porous Media, 114 (2015), pp. 485-503.

Copyright (c) by SIAM. Unauthorized reproduction of this article is prohibited. 